\title{
The Relationship between Quality of Life, Bone Pain, Skin Pruritus and Depression among Patients with Secondary Hyperparathyroidism: A Cross-Sectional Study
}

\author{
Ting Bi, Guangming Cheng, Yufu Tang, Chunhui Wang, Sijia Bai, Shuai Guo, Xiaodong Feng* and \\ Wei Zhang* \\ Department Of Hepatobiliary Surgery, Northern Theater General Hospital, Shenyang, China
}

*Corresponding author: Xiaodong Feng and Wei Zhang, Department of Hepatobiliary Surgery, Northern Theater General Hospital,

Shenyang, China

\section{ARTICLE INFO}

Received: 㓞 November 11, 2021

Published: January 05, 2022

Citation: Ting Bi, Guangming Cheng, Yufu Tang, Xiaodong Feng, Wei Zhang, et al., The Relationship between Quality of Life, Bone Pain, Skin Pruritus and Depression among Patients with Secondary Hyperparathyroidism: A Cross-Sectional Study. Biomed J Sci \& Tech Res 40(5)-2022. BJSTR. MS.ID.006503.

Abbreviations: QOL-BREF: QOL Measurement Scale; SDS: Self-Rating Depression Scale; SEM: Structural Equation Modeling; PTH: Parathyroid Hormone; PTX: Parathyroidectomy; QOL-BREF: The Quality-of-Life Measurement Scale; VAS: The Visual Analog Scale

\begin{abstract}
Objective: To explore the Relationship between Quality of Life, Bone Pain, Skin Pruritus and Depression among Patients with Secondary Hyperparathyroidism.

Methods: A cross-sectional survey was conducted from January 2017 to December 2020 in a third-class hospital in China. The brief table of the QOL measurement scale (QOL-BREF), a self-designed bone pain and skin pruritus scale and the Self-rating Depression Scale (SDS) were used to estimate QOL, bone pain and skin pruritus, and depression, respectively. Pearson's correlation, multiple linear regression analysis and structural equation modeling (SEM) were used for analysis.
\end{abstract}

Results: In SEM, QOL negatively correlated with bone pain ( $\mathrm{r}=-0.509)$, skin pruritus $(\mathrm{r}=-0.517)$ and SDS $(\mathrm{r}=-0.465)$. Bone pain significantly $(\mathrm{P}<0.01)$ and positively correlated with skin pruritus $(\mathrm{r}=0.568)$ and SDS $(\mathrm{r}=0.450)$. Skin pruritus significantly $(\mathrm{P}<0.01)$ and positively correlated with SDS ( $\mathrm{r}=0.426)$. In addition, QOL partially mediated the association between bone pain or skin pruritus and depression (mediation proportion of $48.62 \%$ or $45.08 \%$ ).

Conclusions: SHPT patients have high depression levels and poor QOL due to bone pain and skin pruritus. Bone pain or skin pruritus could increase depression via the intermediary role of QOL.

Keywords: SHPT; QOL; Bone Pain; Skin Pruritus; Depression

\section{Introduction}

SHPT is a common complication of CKD that is characterized by derangements in the homeostasis of calcium, phosphorus and vitamin D [1]. SHPT causes high-turnover bone disease, leading to a decrease in bone mass [2]. Loss of bone mass is mainly manifested by increased cortical bone, with a reduction of bone resorption and mineralized bone on the surface of the cortex due to mineralization defects, with severe bone pain and fracture in severe cases [3]. Pruritus is an unpleasant feeling associated with the urge to scratch; it is a subjective symptom that is caused by many factors and has multiple dimensions [4]. The causes of skin pruritus in patients with SHPT may be related to the increase in parathyroid hormone (PTH), hyperphosphatemia and the accumulation of some medium and large molecular toxins, such as $\beta 2-\mathrm{mg}$, in vivo [5]. Parathyroidectomy (PTX) is the main treatment 
at present and might also reduce calcium-phosphorus product concentrations, PTH and inflammatory factors, thus controlling vascular calcification and relieving bone pain and skin pruritus [6].

Studies have shown that low QOL and severe physical discomfort can affect patients' mental health, especially depressive symptoms [7]. In this study, we hypothesize that there is a mediating effect between bone pain, skin pruritus and depression. QOL is a health concept that comprehensively evaluates the qualitative and quantitative factors such as physical function, psychological, social, economic and emotional factors in a patient's life and is used to evaluate the quality of life in a comprehensive way, which should be based on living standards, but its meaning is more complex and extensive [8]. Kim, et al. research [9] showed that QOL had a strong correlation with pain and depression or anxiety. McIntyre et al. research [10] showed that QOL had a mediating effect between symptoms and depression, which indicated that QOL can indirectly affect depression as a mediating variable. JungHye et al. research [11] proposed a QOL protection model, and the model considered QOL as a protective factor that can regulate the negative impact of risk factors on development outcomes. There are many influencing factors of SDS. At present, the current research mainly focuses on the direct effects of pain and QOL on the occurrence of SDS, but the joint effect of pain and QOL on SDS is not explained. In view of this, this study hypothesize that bone pain/skin pruritus can not only directly affect SDS of SHPT patients, but also indirectly affect SDS by changing the intermediary link of QOL.In this study, it is assumed that QOL plays a regulatory role in the mediation model of bone pain, skin pruritus and depression. In summary, this study hypothesizes that there is a relationship between QOL, bone pain, skin pruritus and depression and that QOL has a mediating effect between bone pain, skin pruritus and depression.

\section{Methods with Statistical Considerations}

\section{Participants and Data Collection}

A cross-sectional study was conducted in a third-class hospital in Liaoning Province, China, from January 2017 to December 2020. The patients with SHPT were aware of the content of the survey before their participation, and on the day before the questionnaires were distributed, with the agreement of the Northern Theater General Hospital ethics committee, we issued informed consent forms to each patient. In this study, the inclusion criteria was all patients needed PTX and signed informed consent and the exclusion criteria was severe cardiac insufficiency and cognitive dysfunction. A total of 325 questionnaires were distributed, and those that were missing more than $20 \%$ of values or had low writing quality were excluded from this survey. In total, 320 questionnaires were considered valid (98.46\% effective response rate).

\section{Measures}

Measurement of QOL: We measured QOL by using the brief table of the quality-of-life measurement scale (WHOQOL-BREF). The WHOQOL-BREF, a self-reported scale, has 24 items, and each item has a 5-point Likert-type scale ranging from 1 to 5 . The total score of the scale ranges from 16 to 80 , and higher scores indicate better QOL. There are four dimensions in this instrument: the physiological dimension, the psychological dimension, the social relation dimension and the environmental dimension [12]. In this study, the Cronbach's $\alpha$ coefficient of internal consistency for the overall scale was 0.857 .

Measurement of Bone Pain and Skin Pruritus: We used the Visual Analog Scale (VAS) to measure bone pain. This scale draws a long horizontal line on the paper; one end of the horizontal line is 0 , indicating no pain, the other end is 10 , indicating severe pain, and the middle region indicates different degrees of bone pain [13]. This method was also used to measure the degree of skin pruritus.

Measurement of Depression: The Self-rating Depression Scale (SDS) is composed of 20 items and 4 dimensions: psychoemotional symptoms, somatic disorders, neuromotor disorders and psychological disorders. The SDS asks about the degree of depression in patients using a 4-point Likert Scale that ranges from never (1 point) to always ( 4 points), and the total score ranges from 20 to 80 [14]. In this study, the Cronbach's $\alpha$ coefficient of internal consistency for the overall scale was 0.842 .

Statistical Analysis: SPSS 21.0 and AMOS 23.0 were used for the statistical analyses. First, single sample K-S test was used to test whether the measurement data conformed to normal distribution. If it was, it was expressed as ' $\mathrm{x} \pm \mathrm{s}$, otherwise it was expressed as median or interquartile range. We conducted descriptive analyses of the sociodemographic variables and Pearson correlation analysis of the other variables. Multiple linear regression analysis was used to test the mediating effect, and the bootstrap method in the AMOS structural equation model was used to further verify the mediating effect. The process of parameter estimations could exclude measurement error [15]. According to related research and theories, a hypothetical relationship model was created and is shown in Figures 1 \& 2. Two SEM models were presented, as follows: QOL is an intermediary variable between bone pain, skin pruritus and depression. The structural path hypothesis is that bone pain and skin pruritus have direct and indirect effects through depression. Furthermore, it shows the results for goodness-offit indexes obtained with SEM for the total sample and individual samples. 


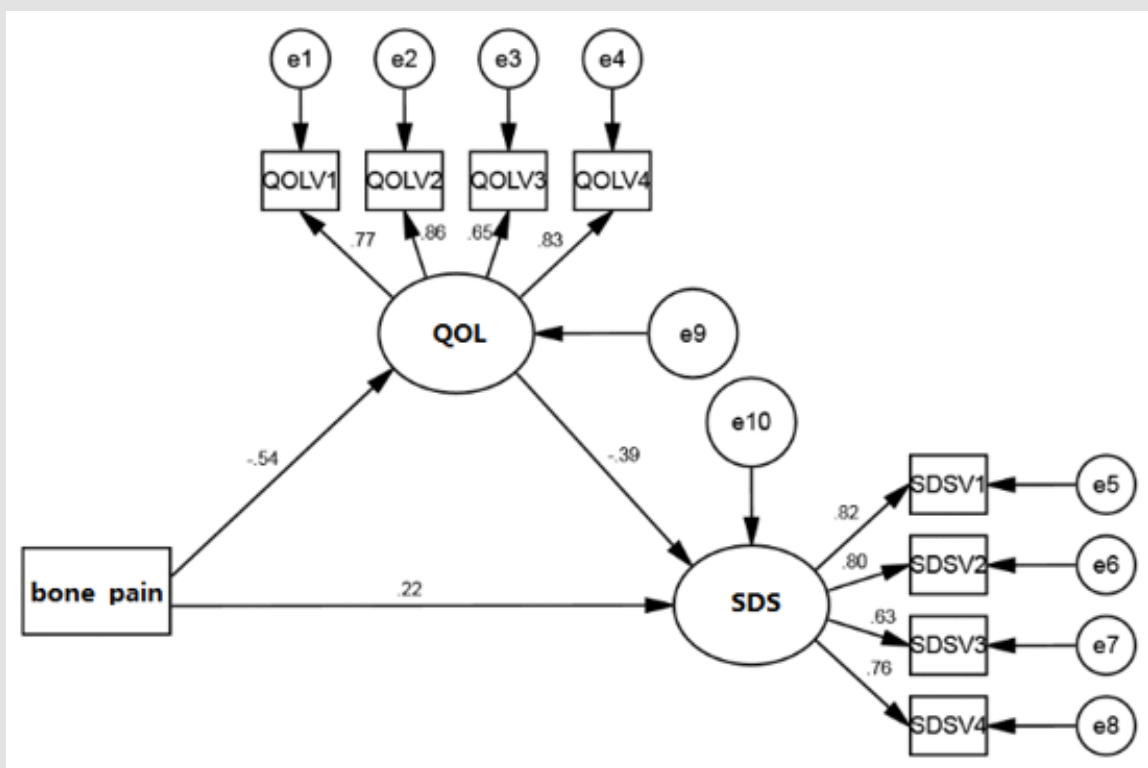

Figure 1: The earth's society: anthropoid setups.

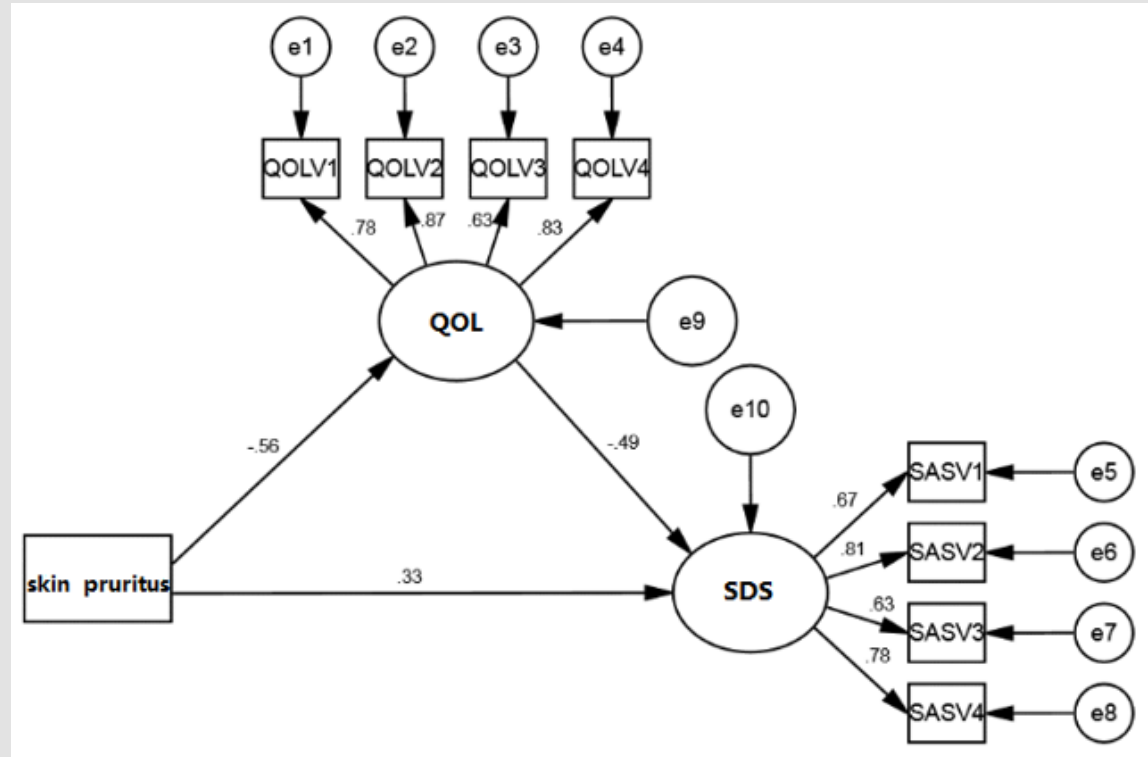

Figure 2: Mediating effect of QOL on skin pruritus and SDS.

\section{Results}

\section{Demographic Characteristics of the Participants}

There were 320 patients with SHPT in this study: 172 (53.8\%) were males, and 148 (46.2\%) were females. 95(92.19\%) had different degrees of forgetfulness,302(94.38\%) had varying degrees of fatigue. The age of the patients ranged from 20 to 70 years. The years of renal failure ranged from 2 to 30 , and the average was $10.71 \pm 4.64$ years. The years of SHPT ranged from 1 to 9 , and the average was $3.07 \pm 2.13$ years.

\section{Descriptive Statistics}

In this study, the measurement data showed a normal distribution, so it was expressed as ` $\mathrm{x} \pm \mathrm{s}$. The QOL score ranged from 25 to 68 , with an average of $41.76 \pm 8.15$. The scores for bone pain and skin pruritus ranged from 1 to 10 , with averages of $5.08 \pm 2.65$ and $3.58 \pm 3.19$, respectively. The years of bone pain ranged from 0 to 10 , and the average was $2.30 \pm 1.95$. The years of skin pruritus ranged from 0 to 8 , and the average was $2.13 \pm 2.05$. The SDS score ranged from 20 to 65 , and the average was $46.04 \pm 8.98$. The 
preoperative PTH ranged from 588 to $2934 \mathrm{pg} / \mathrm{ml}$, and the average was $1729.15 \pm 376.20 \mathrm{pg} / \mathrm{ml}$. The serum calcium ranged from 1.95 to $2.93 \mathrm{mmol} / \mathrm{L}$, and the average was $2.43 \pm 0.19 \mathrm{mmol} / \mathrm{L}$. The serum phosphorus ranged from 1.30 to $3.59 \mathrm{mmol} / \mathrm{L}$, and the average was
$2.38 \pm 0.52 \mathrm{mmol} / \mathrm{L}$. The comparative results of each scale for SHPT patients with different demographic characteristics are shown in Table 1.

Table 1: Descriptive statistics $(\mathrm{N}=320)$.

\begin{tabular}{|c|c|c|c|c|c|c|c|c|}
\hline \multirow{2}{*}{ Variable } & \multicolumn{2}{|c|}{ QOL } & \multicolumn{2}{c|}{ Bone Pain } & \multicolumn{2}{c|}{ Skin Pruritus } & \multicolumn{2}{c|}{ SDS } \\
\cline { 2 - 9 } & $\mathbf{t} / \mathbf{F}$ & $\mathbf{P}$ & $\mathbf{t} / \mathbf{F}$ & $\mathbf{P}$ & $\mathbf{t} / \mathbf{F}$ & $\mathbf{P}$ & $\mathbf{t} / \mathbf{F}$ & -3.156 \\
\hline Gender & 2.066 & 0.04 & -3.313 & 0.001 & -3.3 & 0.001 & 0.002 \\
\hline Age & 1.635 & 0.181 & 2.325 & 0.075 & 0.428 & 0.733 & 2.524 & 0.058 \\
\hline Marital status & 5.783 & 0.001 & 5.407 & 0.001 & 5.786 & 0.001 & 0.309 & 0.819 \\
\hline Occupation & 2.339 & 0.042 & 4.708 & 0.001 & 4.974 & 0.001 & 10.076 & 0.001 \\
\hline Education & 0.688 & 0.601 & 2.589 & 0.037 & 2.807 & 0.026 & 2.949 & 0.02 \\
\hline Income & 2.185 & 0.09 & 1.453 & 0.228 & 2.153 & 0.093 & 5.659 & 0.001 \\
\hline Residential area & 0.207 & 0.892 & 0.337 & 0.798 & 0.463 & 0.708 & 0.955 & 0.414 \\
\hline Insurance & 1.866 & 0.116 & 0.617 & 0.651 & 1.428 & 0.225 & 2.884 & 0.023 \\
\hline
\end{tabular}

\section{Preliminary Correlation Analyses}

First, the correlation analysis of renal replacement therapy (RRT) type, renal failure time, dialysis vintage, duration of SHPT and QOL, bone pain, skin pruritus, SDS showed that renal failure time was significantly $(\mathrm{P}<0.01)$ negatively correlated with $\mathrm{QOL}$ $(r=-0.232)$ and positively correlated with skin pruritus $(r=0.142)$ and SDS ( $r=0.149)$, other variables were not relevant. The Pearson correlation analysis of QOL, bone pain, skin pruritus and SDS is presented in Table 2 . The results showed that QOL was significantly $(\mathrm{P}<0.01)$ and negatively correlated with bone pain $(\mathrm{r}=-0.509)$, skin pruritus ( $\mathrm{r}=-0.517)$ and SDS ( $\mathrm{r}=-0.465)$. Bone pain was significantly $(\mathrm{P}<0.01)$ and positively correlated with skin pruritus $(\mathrm{r}=0.568)$ and SDS $(r=0.450)$. Skin pruritus was significantly $(\mathrm{P}<0.01)$ and positively correlated with SDS $(r=0.426)$.

Table 2: Correlations between the main variables $(\mathrm{N}=320)$.

\begin{tabular}{|c|c|c|c|}
\hline Variable & QOL & Bone pain & Skin pruritus \\
\hline QOL & 1 & & \\
\hline Bone pain & $-0.509^{* *}$ & 1 & \\
\hline Skin pruritus & $-0.517^{* *}$ & $0.568^{* *}$ & 1 \\
\hline SDS & $-0.465^{* *}$ & $0.450^{* *}$ & $0.426^{* *}$ \\
\hline
\end{tabular}

\section{Testing for the Mediation Effect}

The variable was normalized first, and three regression equations were established according to the purpose of this study. Equation 1: depression as the dependent variable and bone pain or skin pruritus as the independent variable; Equation 2: QOL as the dependent variable, with SDS and bone pain or skin pruritus as the independent variable; and Equation 3: SDS as the dependent variable, with QOL and bone pain or skin pruritus as the independent variable. The results showed that bone pain and skin pruritus could significantly predict depression $(\beta=0.485 / 0.375$, $\mathrm{p} \leq 0.001)$ and that bone pain and skin pruritus could significantly predict QOL $(\beta=-0.407 /-0.337, \mathrm{P} \leq 0.001)$. When bone pain or skin pruritus and QOL predicted SDS at the same time, the predictions were significant $(\beta=0.310 / 0.223, \quad p \leq 0.001 ; \beta=-0.429 /-0.450$, $\mathrm{p} \leq 0.001)$. This indicated that the direct or indirect effects of bone pain or skin pruritus on SDS were significant; that is, QOL plays an intermediary role between bone pain or skin pruritus and SDS. The results are shown in Tables $3 \& 4$.

Table 3: The moderating effects of QOL (Part 1).

\begin{tabular}{|c|c|c|c|c|c|c|}
\hline Equation & Dependent variable & Independent variable & $\boldsymbol{\beta}$ & SE & t & P \\
\hline Equation 1 & SDS & Bone pain & 0.485 & 0.054 & 8.984 & 0.001 \\
\hline Equation 2 & QOL & Bone pain & -0.407 & 0.039 & -10.548 & 0.001 \\
\hline Equation 3 & SDS & Bone pain & 0.31 & 0.06 & 5.19 & 0.001 \\
\hline & & QOL & -0.429 & 0.075 & -5.74 & 0.001 \\
\hline
\end{tabular}


Table 4: The moderating effects of QOL (Part 2).

\begin{tabular}{|c|c|c|c|c|c|c|}
\hline Equation & Dependent variable & Independent variable & $\boldsymbol{\beta}$ & SE & t & P \\
\hline Equation 1 & SDS & Skin pruritus & 0.375 & 0.045 & 8.404 & 0.001 \\
\hline Equation 2 & QOL & Skin pruritus & -0.337 & 0.031 & -10.757 & 0.001 \\
\hline Equation 3 & SDS & Skin pruritus & 0.223 & 0.05 & 4.51 & 0.001 \\
\hline & & QOL & -0.45 & 0.076 & -5.929 & 0.001 \\
\hline
\end{tabular}

\section{Verification of the Mediating Effect}

On the basis of multivariate regression analysis, the bootstrap method was used to further test the intermediary effect of QOL. To test the intermediary effect, we needed to establish a structural equation model, and we used AMOS 23.0 to test it. The specific path analysis diagram is shown in Figures $1 \& 2$. The structural equation model fit results showed that CMIN/DF=2.437/1.202 $(<3) ; \quad$ AGFI $=0.928 / 0.965, \quad$ GFI $=0.960 / 0.980, \quad$ TLI $=0.961 / 0.994$,
IFI $=0.973 / 0.996$, and $\quad \mathrm{CFI}=0.973 / 0.996 \quad(>0.9)$; $\quad$ and SRMR=0.042/0.026 and RMSEA=0.067/0.025 $(<0.08)$, indicating that the model fit well. The results showed that the confidence intervals for both the direct and indirect effects of bone pain and skin pruritus on SDS did not reach 0 . The mediating effect model of QOL was established, and the mediating effect was $0.212 / 0.275$, accounting for $48.62 \% / 45.08 \%$ of the total effect. See Tables 5 \& 6 for details.

Table 5: Results for total, indirect and direct effects of bone pain on SDS with QOL as a mediator.

\begin{tabular}{|c|c|c|c|c|c|}
\hline \multirow{2}{*}{\multicolumn{2}{|c|}{ SE }} & \multicolumn{2}{|c|}{ Bias-Corrected 95\%CI } & \multicolumn{2}{|c|}{ Percentile 95\%CI } \\
\hline & & Lower & Upper & Lower & Upper \\
\hline \multicolumn{6}{|c|}{ Total Effect } \\
\hline Bone pain-SDS & 0.436 & 0.279 & 0.585 & 0.276 & 0.581 \\
\hline \multicolumn{6}{|c|}{ Indirect Effect } \\
\hline Bone pain-QOL-SDS & 0.212 & 0.101 & 0.371 & 0.069 & 0.336 \\
\hline \multicolumn{6}{|c|}{ Direct Effect } \\
\hline Bone pain-SDS & 0.224 & 0.053 & 0.43 & 0.061 & 0.438 \\
\hline
\end{tabular}

Table 6: Results for total, indirect and direct effects of skin pruritus on SDS with QOL as a mediator.

\begin{tabular}{|c|c|c|c|c|c|}
\hline \multirow{2}{*}{ SE } & & \multicolumn{2}{|c|}{ Bias-Corrected 95\%CI } & \multicolumn{2}{|c|}{ Percentile95\%CI } \\
\hline & & Lower & Upper & Lower & Upper \\
\hline \multicolumn{6}{|c|}{ Total Effect } \\
\hline Skin pruritus-SDS & 0.61 & 0.435 & 0.795 & 0.433 & 0.792 \\
\hline \multicolumn{6}{|c|}{ Indirect Effect } \\
\hline Skin pruritus-QOL-SDS & 0.275 & 0.188 & 0.378 & 0.183 & 0.374 \\
\hline \multicolumn{6}{|c|}{ Direct Effect } \\
\hline Skin pruritus-SDS & 0.335 & 0.179 & 0.493 & 0.179 & 0.492 \\
\hline
\end{tabular}

\section{Discussion}

\section{The Depression Status of SHPT Patients}

Previous studies have shown that Cinacalet can inhibit PTH in a short time [16], but most patients cannot afford it because of its high price. Meanwhile, previous studies in our department have shown that patients taking Cinacalcet for more than 5 years cannot continue to suppress PTH, but PTX can better suppress PTH, improve symptoms and quality of life. With the transformation of medical models into biological/psychological/social models, the phenomenon of bodily disease accompanied by anxiety and depression has attracted wide attention in the field of medical psychology. The main symptoms of patients with SHPT are depression, memory loss, insomnia, nightmares, etc [17]. However, there are few investigations on QOL and depression psychology at home and abroad. In this study, patients had a high level of depression, with an incidence of $77.81 \%$. The average SDS score was $46.04 \pm 8.98$, which was higher than the norm [18]. This result indicated that the mental health level of patients with SHPT was low, which suggested that more attention should be paid to patients with SHPT in China. The generation of depressive symptoms includes many factors, such as pain, pruritus, occupation, education, 
income, and insurance [19], but in the early stage of depression, relieving discomfort over time and giving timely social or emotional support can largely avoid the occurrence of depression [20]. The SDS score of male patients in this study was higher than that of female patients; however, this is different from Liu's findings [21], which may be related to the fact that men are under more economic pressure and most patients are unable to work due to hemodialysis. There were significant differences in SDS scores among patients with different occupations, education, incomes, and insurance, which may be because the patients with high education and a good job generally enjoy higher medical insurance reimbursement and take Cinacalet, lanthanum carbonate and other drugs to alleviate SHPT [22].

\section{The Status of SHPT Patients' QOL, Bone Pain, and Skin Pruritus}

The vast majority of SHPT patients have skin pruritus, bone pain, electrolyte disorder, coupled with long-term dialysis leading to the destruction of various systems of body, which complicates the screening, such as sleep disturbances, fatigue, loss of energy and poor appetite. However, no related surveys on QOL, bone pain, or skin pruritus in patients with SHPT have been reviewed at home or abroad. In this study, the average QOL score was $41.76 \pm 8.15$, which was lower than the norm [23], and the average score for bone pain was $5.08 \pm 2.65$, which was higher than the norm [24]. This was consistent with Galvez-Sanchez's [7] and Rehman's [25] findings. In this study, skin pruritus had a greater impact on the patient's body than bone pain. PTX can improve the symptoms of bone pain caused by bone deficiency and hypocalcemia.

\section{The Mediating Effect of QOL}

The results of Pearson correlation analysis showed that QOL, bone pain, skin pruritus and depression were significantly correlated with each other, and that bone pain and skin pruritus positively predicted the level of depression. This was consistent with the results obtained by Brophy et al. [26]. and laid the foundation for the subsequent analysis of mediating effects. Notably, regression analysis revealed that there was a partial mediating effect between bone pain, skin pruritus and depression, which provided a basis for the verification of mediating effects. At the same time, some studies also showed that patients with low QOL have a significantly higher risk of depression, and QOL is an important predictor of depression in SHPT patients, which is basically consistent with the results of this study. The bootstrap method was used to further verify the mediation effect. The results showed that a mediating effect of QOL between bone pain, skin pruritus and depression was established, and the mediating effect was $48.62 \% / 45.08 \%$. BI also confirmed that QOL played a role in mediating depression [27]. In clinical work, we should encourage SHPT patients to improve symptoms causing discomfort through PTX in a timely manner, which can greatly reduce depression and improve QOL.

Medical staff should guide family members to actively participate in all aspects of patients' life and treatment, timely grasp the psychological function of patients, and strengthen the care for patients. Encouraging patients to participate in more social activities can buffer the negative effects of various pressures on body and mind, improve sleep quality, reduce depression and other psychological disorders. Therefore, we set up we chat group for SHPT patients to answer the medical problems after discharge online, such as adjusting blood calcium, regular follow-up within the group. The department should carry out the corresponding mental health survey regularly and give timely intervention to the patients with severe pain, pruritus, sleep disorder and depression tendency, and take positive and effective measures to improve mental health level in time. In summary, QOL, bone pain, skin pruritus and depression are closely related in patients with SHPT and there may be a full mediating effect of QOL between bone pain or skin pruritus and depression. It is suggested that alleviating bone pain and skin pruritus in SHPT patients can alleviate the state of depression through the mediating effect of QOL.

\section{Acknowledgment}

Thanks to all of the study participants. An earlier version of the manuscript has been presented as Pre-print to Research Square as per the following source https://www.researchsquare.com/ article/rs-26542/v3.

\section{Funding}

This study was supported by the Foundation for Guidance plan of Liaoning Natural Science(20180551225) and Joint Program of Key R \& D Programs of Liaoning Province(2020JH2/10300168).

\section{Declaration of Conflicting Interests}

The authors declare that they have no competing interests.

\section{Research Ethics and Patient Consent}

The study protocol was approved by the Northern Theater General Hospital ethics committee, we issued informed consent forms to each patient.

\section{Authors' Contributions}

Ting Bi carried out the studies, participated in collecting data, and drafted the manuscript. Xiaodong Feng, Wei Zhang, Guangming Cheng, and Chunhui Wang performed the statistical analysis and participated in its design. Yufu Tang, Sijia Bai, and Shuai Guo participated in acquisition, analysis, or interpretation of data and draft the manuscript. All authors read and approved the final manuscript. 


\section{References}

1. Jacques Rottembourg, Pablo Ureña-Torres, Daniel Toledano, Victor Gueutin, Abdelaziz Hamani, et al. (2019) Factors associated with parathyroid hormone control in haemodialysis patients with secondary hyperparathyroidism treated with cinacalcet in real-world clinical practice: Mimosa study. Clin Kidney J 12: 871-879.

2. Stenio Medeiros Queiroz, Ana Luiza Dias Leite De Andrade, Patrícia Teixeira De Oliveira, Paulo Raphael Leite Maia, Ângelo Giuseppe Roncalli Da Costa Oliveira, et al. (2019) Correlation of Radiomorphometric Indices of the Mandible and Biochemical Parameters in Patients with Secondary Hyperparathyroidism Due to Chronic Kidney Disease. Eur J Dent 13: 303-309.

3. Yifei Ge, Guang Yang, Ningning Wang, Xiaoming Zha, Xiangbao Yu, et al. (2019) Bone metabolism markers and hungry bone syndrome after parathyroidectomy in dialysis patients with secondary hyperparathyroidism. Int Urol Nephrol 51: 1443-1449.

4. Yosipovitch G, Bernhard JD (2013) Clinical practice. Chronic pruritus. N Engl J Med 368: 1625-1634.

5. Qixia Shen, Wenyu Xiang, Sen Ye, Xin Lei, Lefeng Wang, et al. (2019) Plasma metabolite biomarkers related to secondary hyperparathyroidism and parathyroid hormone. J Cell Biochem 120: 15766-15775.

6. Bellorin-Font E, Vasquez-Rios G, Martin KJ (2019) Controversies in the Management of Secondary Hyperparathyroidism in Chronic Kidney Disease. Curr Osteoporos Rep 17: 333-342.

7. Carmen M Galvez-Sánchez, Casandra I Montoro, Stefan Duschek, Gustavo A Reyes Del Paso (2020) Depression and trait-anxiety mediate the influence of clinical pain on health-related quality of life in fibromyalgia. J Affect Disord 265: 486-495.

8. Um-E-Kalsoom (2020) Gender role in anxiety, depression and quality of life in chronic kidney disease patients. Pak J Med Sci 36: 251-254.

9. Do Hyoung Kim, Ji In Park, Jung Pyo Lee, Yong-Lim Kim, Shin-Wook Kang, et al. (2020) The effects of vascular access types on the survival and quality of life and depression in the incident hemodialysis patients. Ren Fail 42: 30-39.

10. Erica McIntyre, Romy Lauche, Jane Frawley, David Sibbritt, Prasuna Reddy, et al. (2019) Physical activity and depression symptoms in women with chronic illness and the mediating role of health-related quality of life. J Affect Disord 252: 294-299.

11. Jung HM, Kim HY (2020) A health-related quality of life model for patients undergoing haemodialysis. J Clin Nurs 29: 613-625.

12. Cardoso J, Almeida T, Ramos C, Sara Sousa, José AA Brito (2019) Bidirectional relationship between sleep disturbances and stress: the role of coping and quality of life [Abstract]. Annals of Medicine 51: 191191.

13. Carles Escalona-Marfil, Andrea Coda, Jorge Ruiz-Moreno, Lluís Miquel Riu-Gispert, Xavier Gironès (2020) Validation of an Electronic Visual Analog Scale mHealth Tool for Acute Pain Assessment: Prospective Cross-Sectional Study. J Med Internet Res 22: e13468.
14. Duan Q, Sheng L (2012) Clinical validity of anxiety and depression selfrating scale. Chinese Journal of mental health 26: 676-679.

15. Huang PH (2017) Asymptotics of AIC, BIC, and RMSEA for Model Selection in Structural Equation Modeling. Psychometrika 82: 407-426.

16. Barbara Meyer-Zehnder, Heidi Albisser Schleger, Sabine Tanner, Valentin Schnurrer, Deborah R Vogt, et al. (2017) How to introduce medical ethics at the bedside - Factors influencing the implementation of an ethical decision-making model. BMC Med Ethics 18: 16.

17. Won Woong Kim, Yumie Rhee, Beom Seok Kim, Kwangsoon Kim, Cho Rok Lee, et al. (2019) Clinical outcomes of parathyroidectomy versus cinacalcet in the clinical management of secondary hyperparathyroidism. Endocr J 66: 881-889.

18. Zhang J, Wu Z, Fang G (2010) Establishment of the national urban norm of the depression scale of the center for flow regulation. Chinese Journal of mental health 24: 139-143.

19. Roger C M Ho, Kwok-Kei Mak, Anna N C Chua, Cyrus S H Ho, Anselm Mak (2013) The effect of severity of depressive disorder on economic burden in a university hospital in Singapore. Expert Rev Pharmacoecon Outcomes Res 13: 549-559.

20. Marroquin B (2011) Interpersonal emotion regulation as a mechanism of social support in depression. Clin Psychol Rev 31: 1276-1290.

21. Chun-Ping Liu, Xiao-Mei Li, Hang-Wei Chen, Jun-Yu Cui, Li-Li Niu, et al. (2011) Depression, anxiety and influencing factors in patients with acute pulmonary embolism. Chin Med J (Engl) 124: 2438-2442.

22. John Cunningham, Geoffrey A Block, Glenn M Chertow, Kerry Cooper, Pieter Evenepoel, et al. (2019) Etelcalcetide Is Effective at All Levels of Severity of Secondary Hyperparathyroidism in Hemodialysis Patients. Kidney Int Rep 4: 987-994.

23. G Liegl, M A Petersen, M Groenvold, N K Aaronson, A Costantini, et al. (2019) Establishing the European Norm for the health-related quality of life domains of the computer-adaptive test EORTC CAT Core. Eur J Cancer 107: 133-141.

24. Mazokopakis EE (2019) Chronic stress and depression are associated with chronic bone pain in Biblical times. Spine J 19: 961

25. Inayat U Rehman, Pauline Sm Lai, Lim S Kun, Learn H Lee, Kok G Chan, et al. (2020) Chronic Kidney Disease-Associated Pruritus and Quality of Life in Malaysian Patients Undergoing Hemodialysis. Ther Apher Dial 24: 17-25.

26. Kyla Brophy, Elmar Brähler, Andreas Hinz, Silke Schmidt, Annett Körner (2020) The role of self-compassion in the relationship between attachment, depression, and quality of life. J Affect Disord 260: 45-52.

27. Bi T, Zhang H (2017) Mediating effect of quality of life on pain and anxiety in middle-aged and elderly glaucoma patients. China public health 33: 509-512. 
ISSN: 2574-1241

DOI: $10.26717 /$ BJSTR.2022.40.006503

Xiaodong Feng, Wei Zhang. Biomed J Sci \& Tech Res

(c) (P) This work is licensed under Creative

Commons Attribution 4.0 License

Submission Link: https://biomedres.us/submit-manuscript.php

$\begin{array}{ll}\text { BIOMEDICAL } & \text { Assets of Publishing with us } \\ \text { RESEARCHES } & \text { - Global archiving of articles } \\ \text { - Immediate, unrestricted online access }\end{array}$

